\section{Mortalidade por doenças cerebrovasculares em idosos e a vacinação contra a influenza: Estado de São Paulo, Brasil, 1980-2012}

\author{
Mortality due to cerebrovascular disease among \\ the elderly and vaccination against influenza: \\ São Paulo State, Brazil, 1980-2012
}

\section{Mortalidad por enfermedades cerebrovasculares en ancianos y la vacunación contra la gripe: Estado de São Paulo, Brasil, 1980-2012}

\section{Resumo}

Este estudo descreve a tendência dos coeficientes de mortalidade por doenças cerebrovasculares (DCbV) em idosos no Estado de São Paulo, Brasil, entre 1980 e 2012, antes e depois das campanhas de vacinação contra a influenza, e identifica pontos de mudanças. Trata-se de um estudo ecológico de série temporal, realizado com dados de óbitos do Sistema de Informações sobre Mortalidade do Ministério da Saúde e dados populacionais do Instituto Brasileiro de Geografia e Estatística. Para análise dos dados, foram utilizados modelos de regressão linear, polinomial e joinpoint regression. Entre 1980 e 2012, foram registrados 480.955 óbitos por DCbV. Os coeficientes médios de mortalidade diminuíram em ambos os sexos para todas as faixas etárias analisadas, com maior redução nas idades mais longevas e no sexo masculino. Observou-se queda significativa na tendência de mortalidade em 1998 para o sexo masculino, na faixa de 60-69 anos (annual percent change - APC = -3\%, IC95\%: -4,3; - 1,6) e para o total dos idosos (APC =-3,8\%, IC95\%: -4, 4; - 3, 1) Considerando-se o período como um todo, não se observaram pontos de mudanças para a faixa de 70-79 (average annual percent change - AAPC $=-3,3 \%$, IC95\%: - 3, 5; -3, 1) e, no sexo masculino, para o grupo $\geq 80$ anos $(A A P C=-2,9 \%$, IC95\%: -3, 1; -2,6). Para o total de idosos, a redução percentual média foi de 3,1\% ao ano $(A A P C=-3,1 \%$, IC95\%: -3,5; -2,7). Os resultados mostraram redução da mortalidade por DCbV no período estudado, com diferentes variações percentuais de queda dos coeficientes. Os achados deste estudo adicionam informações para o debate sobre o possível efeito das campanhas de vacinação na redução da mortalidade por DCbV na população idosa.

Doenças Cardiovasculares; Transtornos Cerebrovasculares; Vacina contra Influenza; Idoso; Mortalidade
Aldiane Gomes de Macedo Bacurau 1

Rosemeire de Olanda Ferraz 1

Maria Rita Donalisio 1

Priscila Maria Stolses Bergamo Francisco 1

doi: 10.1590/0102-311X00145117

\author{
Correspondência \\ A. G. M. Bacurau \\ Departamento de Saúde Coletiva, Faculdade de Ciências \\ Médicas, Universidade Estadual de Campinas. \\ Rua Tessália Vieira de Camargo 126, Cidade Universitária \\ Zeferino Vaz, Campinas, SP 13083-887, Brasil. \\ aldianemacedo@hotmail.com \\ 1 Faculdade de Ciências Médicas, Universidade Estadual de \\ Campinas, Campinas, Brasil.
}




\section{Introdução}

O rápido e heterogêneo envelhecimento populacional brasileiro produz uma série de desafios para o sistema de saúde, especialmente com demandas por serviços e políticas adequadas às necessidades da população idosa 1 . Apesar do processo de envelhecimento não estar necessariamente relacionado a doenças e incapacidades, os idosos apresentam alta prevalência de doenças crônicas não transmissíveis (DCNT) 2. No Brasil, o aumento na prevalência das DCNT constitui um problema de saúde pública de grande magnitude, com destaque para as doenças cardiovasculares 3 .

As doenças cardiovasculares (DCV) representam a principal causa de morte, tanto nos países desenvolvidos como naqueles em desenvolvimento, e correspondem a cerca de 30\% dos óbitos na população mundial. Segundo a Organização Mundial da Saúde (OMS), apesar da diminuição da mortalidade em algumas regiões, mais de 75\% dos óbitos por essas causas ocorrem em países de baixa ou média renda, e cerca de $80 \%$ das mortes são devidas a infarto agudo do miocárdio (IAM) e acidentes vasculares cerebrais (AVC), o que destaca o impacto das doenças isquêmicas do coração (DIC) e doenças cerebrovasculares (DCbV) na mortalidade por DCV 4.

É importante considerar que os fatores de risco para as doenças cardiovasculares são mais prevalentes e mais graves com o aumento da idade e, apesar de muitos idosos não apresentarem doenças evidentes, frequentemente possuem comorbidades, doenças subclínicas, alterações funcionais e anatômicas que agem modificando a estrutura cardiovascular, facilitando a atuação dos mecanismos fisiopatológicos da doença 5 .

No Brasil, as DCV foram responsáveis por 34\% dos óbitos registrados na população idosa (60 anos ou mais), em 2014. No Estado de São Paulo, no mesmo ano, essa proporção foi de 33,5\%, destacandose as DIC e as DCbV como as causas de óbitos por DCV mais comuns nos idosos, 34\% e 26,9\%, respectivamente (Departamento de Informática do SUS. Estatísticas vitais. http://www2.datasus.gov.br/ DATASUS/index.php?area=0205, acessado em 05/Abr/2017). Além de ser a principal causa de morte, as DCV também são responsáveis por elevados custos de internação e de seguimento ambulatorial nos idosos 6. A taxa de hospitalização por DCV naqueles com 60 anos ou mais no país é nove vezes maior em relação à população adulta (20-59 anos), e os gastos com as internações hospitalares dos idosos, devido a esse agravo, são oito vezes mais elevados em relação à população adulta 7 .

Estudos sugerem que a infecção pelo vírus da influenza está associada a um aumento de complicações, hospitalizações e mortes por DCV 8,9. O Centers for Disease Control and Prevention (CDC; Estados Unidos) destaca que a infecção prévia por influenza é fator de risco para mortes causadas por IAM, doença vascular e diabetes, no entanto, como essas mortes não são consideradas junto aos óbitos por pneumonia e influenza, o impacto da influenza pode ser subestimado 10 .

Como estratégia para redução das internações, complicações e óbitos associados à infecção pela influenza, o Ministério da Saúde recomenda uma dose anual da vacina para os grupos considerados de risco, incluindo os idosos e indivíduos portadores de doenças crônicas 11,12. Desde o ano 2006, as diretrizes da American Heart Association e do American College of Cardiology recomendam uma dose anual da vacina para os pacientes com doença aterosclerótica em geral 13. A Sociedade Brasileira de Cardiologia (SBC) também indica a vacina contra influenza para pacientes com insuficiência cardíaca 14

As campanhas de vacinação contra gripe tiveram início no Brasil no ano 1999. Inicialmente a vacina foi disponibilizada para os indivíduos com idade $\geq 65$ anos e, a partir do ano 2000, para a população com idade $\geq 60$ anos. No entanto, na cidade de São Paulo, a vacinação dos idosos foi instituída após a aprovação da Lei Municipal no 12.326/97 e, em junho de 1998, foi aprovada a Lei no 10.003/98, que estendeu a vacinação dos idosos para todo o Estado de São Paulo 15.

Existem vários estudos desenvolvidos no Brasil sobre hospitalizações e óbitos por causas respiratórias em idosos, que indicam a redução ou estabilidade desses indicadores após o início da vacinação contra a influenza 16,17. Os efeitos da vacina em indivíduos com DCV têm sido motivo de investigação em estudos observacionais que, apesar de não apresentarem resultados unânimes, indicam efeito protetor na maioria deles, sobretudo em relação aos desfechos cardíacos 18,19,20.

O objetivo deste estudo é descrever a tendência dos coeficientes de mortalidade por doenças cerebrovasculares em idosos no Estado de São Paulo, entre 1980 e 2012, antes e depois das campanhas de vacinação contra influenza e identificar pontos de mudança na tendência de mortalidade. 


\section{Material e métodos}

\section{Desenho do estudo}

Trata-se de um estudo ecológico de série temporal da tendência dos coeficientes de mortalidade por DCbV na população idosa do Estado de São Paulo, no período de 1980 a 2012. Considerou-se a definição de idoso da OMS para países em desenvolvimento (pessoas com 60 anos ou mais de idade).

As informações sobre os óbitos foram coletadas na base de dados do Sistema de Informações sobre Mortalidade (SIM) do Ministério da Saúde, disponíveis no site do Departamento de Informática do Sistema Único de Saúde (DATASUS; http://www.datasus.gov.br). Os óbitos por causas externas foram utilizados como controle negativo para a comparação da tendência dos coeficientes de mortalidade. Todos os óbitos registrados da população idosa residente no Estado de São Paulo que tiveram como causa básica as $\mathrm{DCbV}$ e causas externas foram selecionados e classificados de acordo com 9a revisão da Classificação Internacional de Doenças (CID-9) para o período de 1980 a 1995 (capítulo VII, códigos de 430 a 438 para as DCbV e capítulo XVII, códigos de E47 a E56 para as causas externas) e de acordo com a 10a revisão (CID-10) para o período de 1996 a 2012 (capítulo IX, códigos I60 a I69 para as DCbV e capítulo XX, códigos de V01 a Y98 para as causas externas), e foram estratificados por sexo e faixas etárias (60-69, 70-79 e $\geq 80$ anos). Os dados da população idosa residente no Estado de São Paulo, por sexo e faixas etárias, foram obtidos por meio das informações demográficas do Instituto Brasileiro de Geografia e Estatística (IBGE), que são informações baseadas nos censos demográficos e nas estimativas populacionais intercensitárias (1980 a 2012).

\section{Análise dos dados}

Os dados sobre os óbitos e a população residente foram exportados para planilhas no Microsoft Excel (https://products.office.com/), nas quais foram calculados os coeficientes anuais de mortalidade por 10 mil habitantes. Para análise, utilizaram-se os coeficientes padronizados por idade (pelo método direto, considerando-se como população padrão a do Censo Demográfico de 2010. http://www.ibge. gov.br) e os coeficientes específicos segundo sexo e faixas etárias. Para corrigir eventuais diferenças nas séries dos coeficientes de mortalidade, decorrentes da transição da CID-9 para a CID-10, foi aplicada a razão de comparabilidade proposta por Anderson et al. 21.

Para analisar a tendência da mortalidade por DCbV, utilizaram-se modelos de regressão linear e polinomial. Inicialmente foi realizada a verificação dos pressupostos de normalidade, homocedasticidade e não autocorrelação para garantir a validade das estimativas obtidas 22 . Os coeficientes de mortalidade foram considerados como variável dependente $(\mathrm{Y})$, e os anos calendários como variável independente $(\mathrm{X})$. Para atenuar a correlação serial de tempo, empregou-se a variável ano centrado (X - ponto médio da série histórica), ou seja, ano 1996 23. Entre os modelos selecionados com base no coeficiente de determinação $\left(\mathrm{R}^{2}\right)$, utilizou-se o mais parcimonioso ${ }^{24}$. Na análise da tendência dos coeficientes de mortalidade por DCbV (regressão linear e polinomial), foram considerados dois momentos no período de 1980 a 2012, denominados de antes (1980 a 1997) e depois (1998 a 2012) das campanhas de vacinação contra a influenza no Estado de São Paulo.

Também foi analisada a tendência com o uso dos modelos de regressão segmentada (joinpoint regression) para identificar pontos de mudanças estatisticamente significativas e a variação percentual anual dos coeficientes de mortalidade entre 1980 e 2012. Joinpoint regression é uma técnica de modelagem estatística que tenta explicar a relação entre duas variáveis por meio de retas de regressão. Os pontos que unem essas retas são chamados de pontos de junção (ou pontos de inflexão). Esse modelo assume uma tendência linear entre esses pontos e possui os mesmos pressupostos da regressão linear, exceto o de homocedasticidade e não autocorrelação, no entanto o software permite incorporar essas condições quando violadas, ou seja, ajusta um modelo de regressão ponderado 25,26. Os pressupostos de normalidade, homocedasticidade e não autocorrelação foram verificados pelos testes de Shapiro \& Wilk 27, White 28 e Godfrey 29 , respectivamente.

A análise possibilita o ajuste de dados de uma série a partir do número mínimo de joinpoints e testa se a inclusão de um ou mais pontos é significativa (assim, zero joinpoint indica uma reta de regressão sem pontos de inflexão). A seleção do número de pontos de junção para obter o modelo significativo 
foi realizada utilizando-se as configurações padrão do software pelo método Grid Search (permite que os joinpoints ocorram exatamente nos anos observados), e os testes de significância para escolha do melhor modelo basearam-se no método de permutação de Monte Carlo 25,26,30,31. Com isso, foram estimadas as variações percentuais anuais dos coeficientes de mortalidade para os períodos delimitados pelos pontos de inflexão, representadas pela annual percent change (APC), assim como as variações percentuais anuais médias da tendência ao longo do período (entre 1980 e 2012), representadas pela average annual percent change (AAPC), e seus respectivos intervalos de confiança 25,26.

Para todos os testes estatísticos realizados neste estudo, foi considerado um nível de significância de 5\% e intervalos de 95\% de confiança (IC95\%). Os softwares utilizados foram o Microsoft Excel, o SPSS (https://www.ibm.com/) versão 21, o Stata (https://www.stata.com) versão 12 e o Joinpoint Regression Program (https://surveillance.cancer.gov/joinpoint/) versão 4.3.1.0.

Por se tratar de um estudo realizado com dados secundários de domínio público, foi apresentado ao Comitê de Ética em Pesquisa (CEP) da Universidade Estadual de Campinas (Unicamp) e recebeu dispensa para apreciação e/ou aprovação do Comitê de Ética em Pesquisas com Seres Humanos, por meio do ofício CEP/PRP/no 001/2016.

\section{Resultados}

Entre 1980 e 2012, foram registrados 480.955 óbitos por DCbV nos idosos no Estado de São Paulo. A tendência de mortalidade foi de queda para ambos os sexos. Observaram-se maiores coeficientes de mortalidade por DCbV na população idosa masculina. Os coeficientes de mortalidade por causas externas se mantiveram constantes no período (Figura 1). Para os idosos do sexo masculino, o coeficiente médio de mortalidade por $\operatorname{DCbV}\left(\hat{\beta}_{0}\right)$ no período foi de 69,32 óbitos por 10 mil idosos, com decréscimo linear constante $\left(\hat{\beta}_{1}\right)$ de $-2,11$ óbitos ao ano. No sexo feminino, o coeficiente médio foi de 55,05 óbitos por 10 mil idosas, com redução não constante $\left(\hat{\beta}_{2}\right)$ de $-1,79$ óbitos ao ano (Tabela 1 ).

Houve diminuição dos coeficientes médios de mortalidade $\left(\hat{\beta}_{0}\right)$ considerando-se os períodos analisados (antes e depois das campanhas de vacinação contra a influenza no estado), que passaram de 85,21 (IC95\%: 83,51; 86,91) para 50,24 (IC95\%: 48,63; 51,85) óbitos por 10 mil/idosos e de 67,39 (IC95\%: 65,24; 69,55) para 40,53 (IC95\%: 39,07; 41,99) óbitos por 10 mil/idosas. Para a população idosa total, os coeficientes médios de mortalidade foram reduzidos de 72,14 (IC95\%: 68,91; 75,38) para 44,80 (IC95\%: 43,29; 46,30) óbitos por 10 mil/habitantes (Tabela 1).

Os coeficientes médios de mortalidade por DCbV aumentaram progressivamente com a idade e mais que duplicaram de uma faixa etária para a seguinte. No sexo feminino, para as idosas com idade $\geq 80$ anos, os coeficientes foram cerca de três vezes os observados na faixa etária de 70-79 anos (Tabela 1 e Figura 2). Observaram-se reduções do coeficiente angular $\left(\hat{\beta}_{1}\right)$ no período após a vacinação para o conjunto dos idosos e também segundo sexo, no entanto, não foram estatisticamente significativas, conforme verificado pelos intervalos de 95\% de confiança (IC95\%) (Tabela 1).

A Tabela 2 apresenta os resultados da análise da tendência de mortalidade por DCbV, entre 1980 e 2012, pela regressão segmentada (joinpoint regression). No sexo masculino, para o conjunto dos idosos, a tendência apresentou apenas um ponto de inflexão ao longo do período, com tendência decrescente significativa entre 1980 e 1995 (APC = -2,5\%, IC95\%: -2,9; -2,0) e redução mais acentuada a partir de 1995 até 2012 (APC = -3,6\%, IC95\%: -4,0; -3,3). No sexo feminino, houve dois pontos de inflexão, os quais evidenciaram uma tendência de queda significativa entre 1980 e 1992 (APC = -3,4\%, IC95\%: $-4,2 ;-2,6)$, discreto aumento não significativo entre 1992 e 1995 e tendência decrescente significativa de 1995 a 2012 com APC de -3,9\% (IC95\%: -4,3; -3,5).

$\mathrm{Na}$ análise por sexo e faixas etárias, observou-se, para a tendência entre 1980 e 2012, redução significativa da mortalidade em ambos os sexos, com AAPC de -3,1\% (IC95\%: -3,4; -2,8) no sexo masculino e -3,4\% (IC95\%: -4,9; -1,8) no feminino. Para o sexo masculino, na faixa etária de 60-69 anos, houve quatro pontos de inflexão na tendência, mas apenas duas tendências foram estatisticamente significativas: as reduções entre 1980 e 1995 (APC =-2,3\%) e entre 1998 e 2005 (APC =-3,0\%). Já no sexo feminino, para a faixa etária de 60-69 anos, todas as mudanças ocorridas na tendência indicaram reduções estatisticamente significativas, com pontos de mudanças em 1986 e 1996 e AAPC de -3,4\% (IC95\%: -3,8; -3,0). Não houve pontos de inflexão na tendência para a faixa de 70-79 anos, em ambos 


\section{Figura 1}

Tendência dos coeficientes de mortalidade por doenças cerebrovasculares (DCbV) e por causas externas, por 10 mil habitantes, da população idosa, segundo sexo. Estado de São Paulo, Brasil, 1980-2012.

1a) Por DCbV

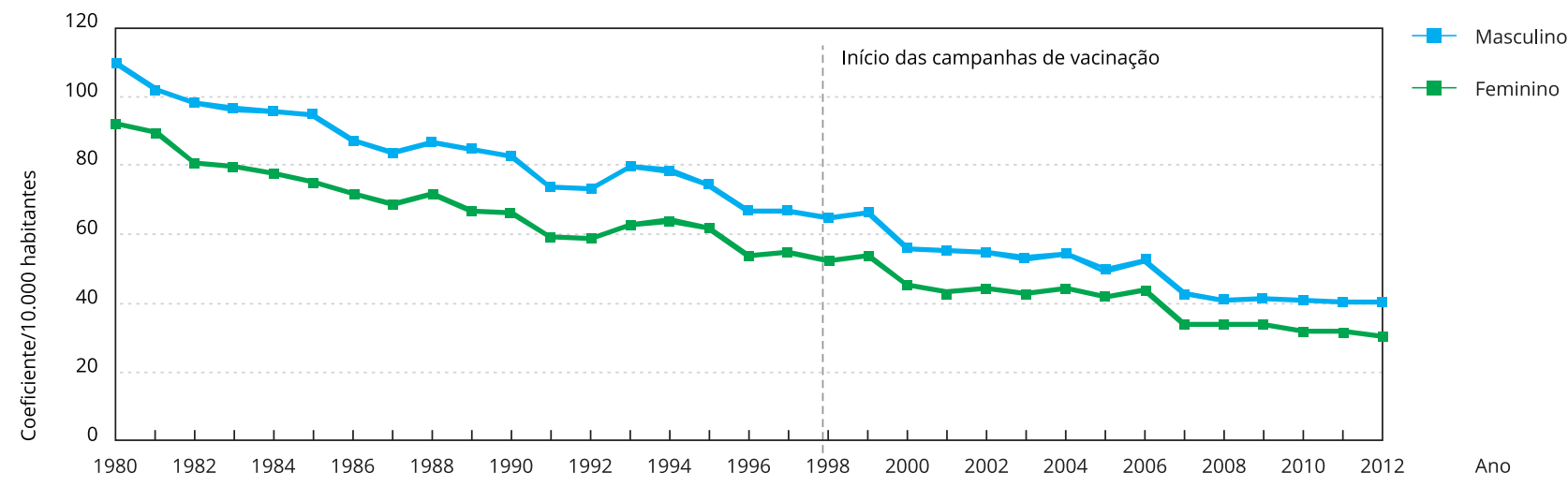

1b) Por causas externas

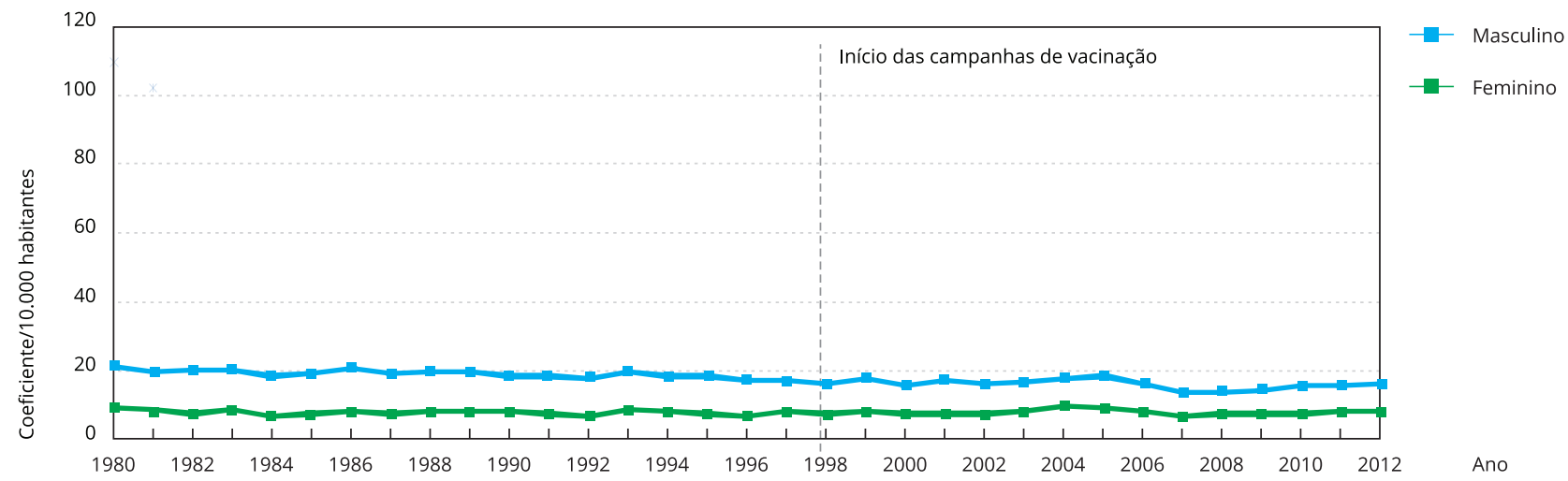

os sexos. Nos mais longevos ( $\geq 80$ anos), não se observaram mudanças na tendência para o sexo masculino (AAPC = -2,9\%, IC95\%: -3,1;-2,6), mas no sexo feminino houve cinco pontos de inflexão, com duas tendências de queda estatisticamente significativas: entre 1980-1991 (APC = -3,4\%) e 1994-2001 $(\mathrm{APC}=-5,4 \%)$ (Tabela 2).

Considerando-se o total dos idosos, observou-se mudança estatisticamente significativa na tendência em 1998, de modo que a redução dos coeficientes de mortalidade que era de 2,6\% (IC95\%: -3,0; $-2,2)$ passou a ser mais acentuada a partir daquele ano (APC $=-3,8 \%$, IC95\%: -4,4; -3,1) (Tabela 2 ). 
Tabela 1

Modelos de regressão linear e polinomial ajustados para os coeficientes de mortalidade por doenças cerebrovasculares (DCbV) por 10 mil habitantes, segundo sexo e faixas etárias. Estado de São Paulo, Brasil, 1980-2012.

\begin{tabular}{lcccccccc}
\hline Sexo & $\hat{\boldsymbol{\beta}}_{\mathbf{0}}$ & IC95\% & $\hat{\boldsymbol{\beta}}_{\mathbf{1}}$ & IC95\% & $\hat{\boldsymbol{\beta}}_{\mathbf{2}}$ & IC95\% & Valor de $\mathbf{p}$ & $\mathbf{R}^{\mathbf{2}}$ \\
\hline Masculino & & & & & & & \\
60-69 anos & 34,47 & 33,$99 ; 34,96$ & $-1,10$ & $-1,15 ;-1,06$ & - & - & $<0,001$ & 0,98 \\
70-79 anos & 84,77 & 82,$55 ; 86,00$ & $-2,70$ & $-2,85 ;-2,54$ & 0,02 & 0,$003 ; 0,039$ & $<0,001$ & 0,98 \\
$\geq 80$ anos & 198,39 & 194,$03 ; 202,75$ & $-5,56$ & $-6,02 ;-5,10$ & - & - & $<0,001$ & 0,95 \\
Total & 69,32 & 68,$18 ; 70,46$ & $-2,11$ & $-2,22 ;-1,98$ & - & - & $<0,001$ & 0,98 \\
Antes * & 85,21 & 83,$51 ; 86,91$ & $-2,20$ & $-2,53 ;-1,87$ & - & - & $<0,001$ & 0,93 \\
Depois ** & 50,24 & 48,$63 ; 51,85$ & $-1,83$ & $-2,21 ;-1,46$ & - & - & $<0,001$ & 0,90 \\
Feminino & & & & & & & & \\
60-69 anos & 18,66 & 18,$23 ; 19,09$ & $-0,60$ & $-0,63 ;-0,57$ & 0,006 & 0,$002 ; 0,009$ & $<0,001$ & 0,98 \\
70-79 anos & 55,76 & 54,$24 ; 57,29$ & $-2,06$ & $-2,17 ;-1,96$ & 0,03 & 0,$015 ; 0,040$ & $<0,001$ & 0,98 \\
$\geq 80$ anos & 177,72 & 173,$45 ; 181,99$ & $-5,20$ & $-5,64 ;-4,75$ & - & - & $<0,001$ & 0,95 \\
Total & 55,05 & 53,$45 ; 56,65$ & $-1,79$ & $-1,90 ;-1,67$ & 0,02 & 0,$003 ; 0,029$ & $<0,001$ & 0,97 \\
Antes * & 67,39 & 65,$24 ; 69,55$ & $-1,97$ & $-2,25 ;-1,69$ & 0,09 & 0,$029 ; 0,149$ & $<0,001$ & 0,94 \\
Depois ** & 40,53 & 39,$07 ; 41,99$ & $-1,57$ & $-1,91 ;-1,23$ & - & - & $<0,001$ & 0,89 \\
Total & & & & & & & & \\
60-69 anos & 25,52 & 24,$94 ; 26,11$ & $-0,81$ & $-0,86 ;-0,77$ & 0,01 & $<0,001 ; 0,009$ & $<0,001$ & 0,98 \\
70-79 anos & 67,24 & 64,$97 ; 69,51$ & $-2,26$ & $-2,42 ;-2,10$ & 0,03 & 0,$007 ; 0,045$ & $<0,001$ & 0,97 \\
$\geq 80$ anos & 181,58 & 176,$28 ; 186,87$ & $-4,99$ & $-5,54 ;-4,43$ & - & - & $<0,001$ & 0,91 \\
Total & 61,22 & 59,$79 ; 62,65$ & $-1,85$ & $-1,99 ;-1,69$ & - & - & $<0,001$ & 0,95 \\
Antes * & 72,14 & 68,$91 ; 75,38$ & $-2,03$ & $-2,45 ;-1,62$ & 0,10 & 0,$013 ; 0,192$ & $<0,001$ & 0,88 \\
Depois ** & 44,80 & 43,$29 ; 46,30$ & $-1,70$ & $-2,04 ;-1,35$ & - & - & $<0,001$ & 0,89 \\
\hline
\end{tabular}

IC95\%: intervalo de 95\% de confiança; R2: coeficiente de determinação.

Nota: $\hat{\beta}_{0}$ : coeficiente médio do período (por 10 mil habitantes); $\hat{\beta}_{1}$ : decréscimo médio anual; $\hat{\beta}_{2}$ : coeficiente de efeito quadrático. Valor de p: nível descritivo do teste.

* Período de 1980 a 1997;

** Período de 1998 a 2012

\section{Discussão}

Este estudo verificou tendência de queda da mortalidade por DCbV, tanto para os homens quanto para as mulheres idosas no período estudado. Observaram-se diferentes variações percentuais de redução dos coeficientes. Particularmente para os idosos do sexo masculino, com idade entre 60-69 anos e para o conjunto dos idosos, houve mudança na tendência em 1998.

A redução da mortalidade dos idosos por DCbV verificada segue a tendência de queda da mortalidade por DCV observada nas últimas décadas para o Brasil e a maioria das suas regiões, para todas as faixas etárias 32,33. Apesar de as regiões Sudeste e Sul do país apresentarem os maiores coeficientes de mortalidade por DCbV, observam-se, entre 1980 e 2012, maiores reduções da mortalidade nessas duas regiões (-61,99\% e -55,49\%, respectivamente) 32 .

Essa redução da mortalidade pode ser reflexo de mudanças de comportamentos e controle dos principais fatores de risco para as DCbV, como tabagismo, dislipidemias, hipertensão arterial, alimentação inadequada, diabetes, sedentarismo, entre outros 34,35. Além disso, o maior acesso aos serviços de saúde, a expansão das ações de promoção da saúde e prevenção de agravos, bem como as melhorias nas condições socioeconômicas da população têm contribuído para explicar, mesmo que parcialmente, essa diminuição da mortalidade por DCbV na população adulta e idosa 3,36,37. Ademais, estudos mostram que o controle e a prevenção das DCV, e consequentemente das DCbV, tendem a ser mais efetivos nas regiões mais desenvolvidas 35,38 . 


\section{Figura 2}

Tendência dos coeficientes de mortalidade por doenças cerebrovasculares (DCbV) e por causas externas, por 10 mil habitantes, da população idosa, segundo faixas etárias. Estado de São Paulo, Brasil, 1980-2012.

2a) 60-69 anos

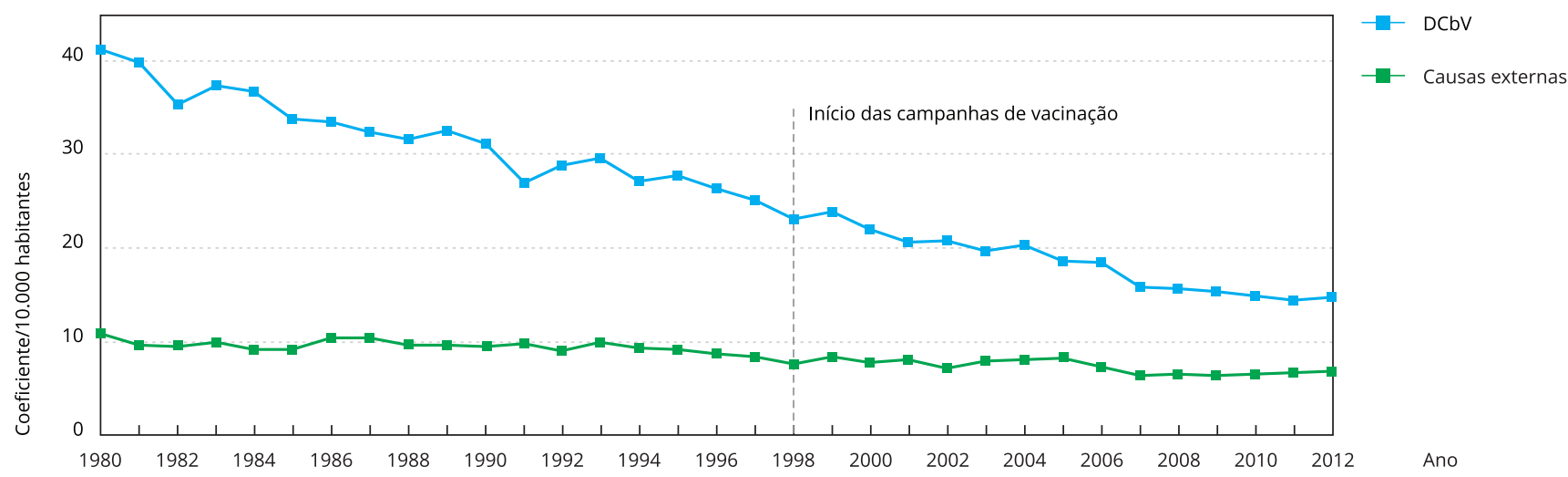

2b) $70-79$ anos

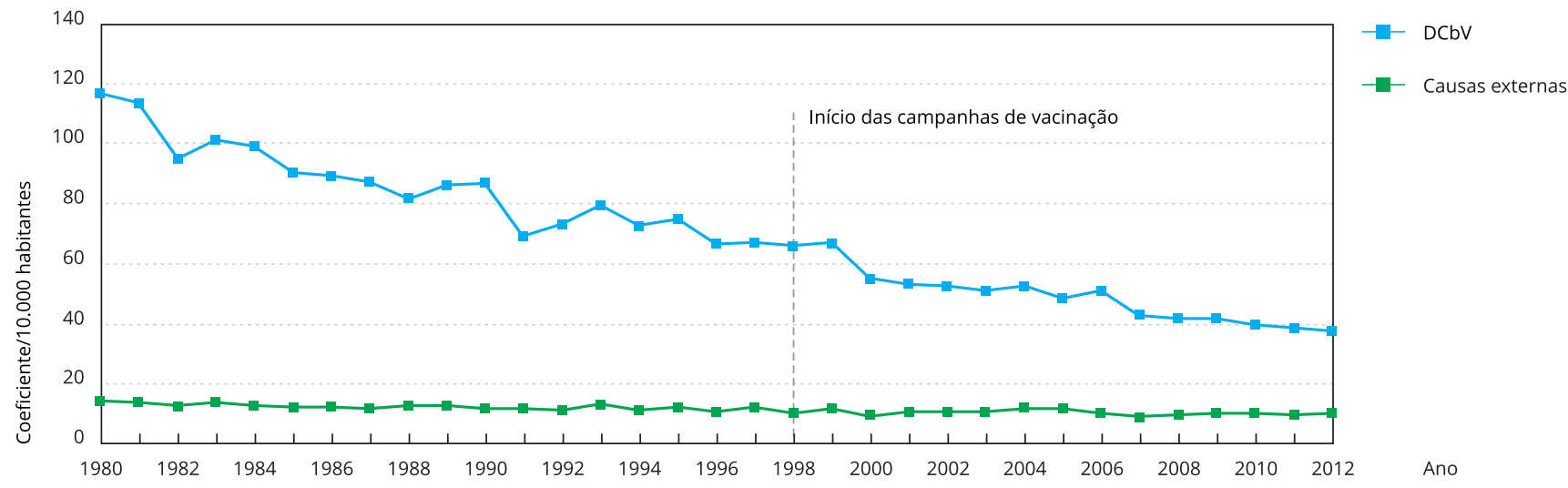

2c) $\geq 80$ anos

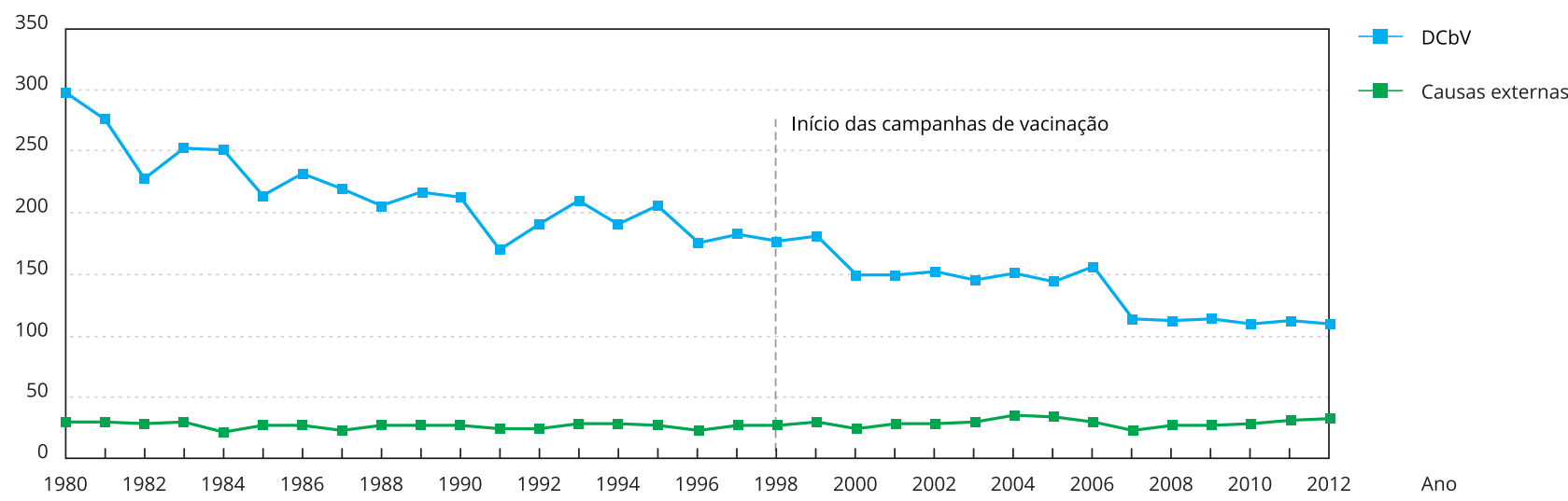




\section{Tabela 2}

Estimativas das variações percentuais dos coeficientes de mortalidade por doenças cerebrovasculares (DCbV) por 10 mil habitantes em idosos, segundo sexo e faixas etárias: joinpoint regression. Estado de São Paulo, Brasil, 1980-2012.

\begin{tabular}{|c|c|c|c|c|}
\hline Sexo/Faixa etária (anos)/Períodos & APC & IC95\% & AAPC & IC95\% \\
\hline Masculino & & & $-3,1 *$ & $-4,5 ;-1,6$ \\
\hline \multicolumn{5}{|l|}{$60-69$} \\
\hline 1980-1995 & $-2,3 *$ & $-2,6 ;-1,9$ & & \\
\hline 1995-1998 & $-6,1$ & $-16,2 ; 5,3$ & & \\
\hline $1998-2005$ & $-3,0$ * & $-4,3 ;-1,6$ & & \\
\hline $2005-2008$ & $-7,0$ & $-17,0 ; 4,2$ & & \\
\hline $2008-2012$ & $-1,0$ & $-3,8 ; 1,8$ & & \\
\hline $70-79$ & & & $-3,2$ * & $-3,4 ;-3,0$ \\
\hline $1980-2012$ & $-3,2 *$ & $-3,4 ;-3,0$ & & \\
\hline$\geq 80$ & & & $-2,9 *$ & $-3,1 ;-2,6$ \\
\hline $1980-2012$ & $-2,9$ * & $-3,1 ;-2,6$ & & \\
\hline Total & & & $-3,1 *$ & $-3,4 ;-2,8$ \\
\hline 1980-1995 & $-2,5 *$ & $-2,9 ;-2,0$ & & \\
\hline $1995-2012$ & $-3,6$ * & $-4,0 ;-3,3$ & & \\
\hline \multicolumn{5}{|l|}{ Feminino } \\
\hline $60-69$ & & & $-3,4$ * & $-3,8 ;-3,0$ \\
\hline $1980-1986$ & $-4,0$ * & $-5,5 ;-2,4$ & & \\
\hline 1986-1996 & $-2,2 *$ & $-3,1 ;-1,4$ & & \\
\hline $1996-2012$ & $-3,8$ * & $-4,1 ;-3,5$ & & \\
\hline $70-79$ & & & $-3,6$ * & $-3,8 ;-3,4$ \\
\hline $1980-2012$ & $-3,6$ * & $-3,8 ;-, 34$ & & \\
\hline$\geq 80$ & & & $-3,1 *$ & $-5,0 ;-1,1$ \\
\hline 1980-1991 & $-3,4$ * & $-4,1 ;-2,7$ & & \\
\hline 1991-1994 & 3,1 & $-10,9 ; 19,3$ & & \\
\hline $1994-2001$ & $-5,4$ * & $-7,1 ;-3,6$ & & \\
\hline $2001-2005$ & 1,1 & $-5,1 ; 7,6$ & & \\
\hline $2005-2008$ & $-10,2$ & $-22,4 ; 4,0$ & & \\
\hline $2008-2012$ & $-1,2$ & $-4,8 ; 2,6$ & & \\
\hline Total & & & $-3,4$ * & $-4,9 ;-1,8$ \\
\hline 1980-1992 & $-3,4$ * & $-4,2 ;-2,6$ & & \\
\hline 1992-1995 & 0,3 & $-15,7 ; 19,3$ & & \\
\hline $1995-2012$ & $-3,9$ * & $-4,3 ;-3,5$ & & \\
\hline \multicolumn{5}{|l|}{ Total } \\
\hline $60-69$ & & & $-3,2 *$ & $-3,5 ;-3,0$ \\
\hline $1980-1669$ & $-2,6$ * & $-2,9 ;-2,2$ & & \\
\hline $1996-2012$ & $-3,9$ * & $-4,2 ;-3,5$ & & \\
\hline $70-79$ & & & $-3,3$ * & $-3,5 ;-3,1$ \\
\hline $1980-2012$ & $-3,3$ * & $-3,5 ;-3,1$ & & \\
\hline$\geq 80$ & & & $-3,0$ * & $-3,5 ;-2,4$ \\
\hline $1980-2004$ & $-2,5^{*}$ & $-2,8 ;-2,1$ & & \\
\hline $2004-2012$ & $-4,4$ * & $-6,4 ;-2,4$ & & \\
\hline Total & & & $-3,1 *$ & $-3,5 ;-2,7$ \\
\hline $1980-1998$ & $-2,6$ * & $-3,0 ;-2,2$ & & \\
\hline $1998-2012$ & $-3,8$ * & $-4,4 ;-3,1$ & & \\
\hline
\end{tabular}

AAPC: average annual percent change (variação percentual anual média); APC: annual percent change (variação percentual anual); IC95\%: intervalo de $95 \%$ de confiança.

Nota: AAPC para o período como um todo (1980 a 2012).

* Valor estatisticamente significativo em relação a hipótese de ausência de tendência temporal para cada segmento $(p<0,001)$. 
Apesar da queda da mortalidade nas regiões mais desenvolvidas do país, as DCbV, principalmente as DIC e as DCbV, ainda são a principal causa de morte no país 36. As estratégias para identificação, prevenção e controle dos fatores de risco para morbidade e mortalidade associadas a essas causas devem continuar sendo uma prioridade de saúde pública 37.

A elevação no número de mortes e eventos cardiovasculares durante as epidemias de gripe tem sido reportada na literatura 39,40,41. Estudos em pacientes com doenças cardíacas crônicas indicam que a influenza é fator de risco para desfechos cardiovasculares, pois pode ocasionar miocardite, arritmias cardíacas e IAM, acarretando elevado número de complicações e óbitos, mesmo em indivíduos previamente saudáveis 9,40 .

Várias hipóteses sobre os mecanismos para associação da infecção pelo vírus influenza e eventos cardiovasculares têm sido descritas na literatura 39,42. Naghavi et al. 43 relacionam alguns desses mecanismos, por exemplo, quando o organismo desencadeia resposta imune que causa inflamação endotelial com alteração de sua função e instabilidade de placas ateroscleróticas. Pode ocorrer a estimulação de citocinas e a proliferação de macrófagos que ativam metaloproteinases que também induzem a ruptura da placa aterosclerótica. É importante considerar que esses mecanismos podem causar a oclusão do fluxo sanguíneo e, consequentemente, eventos como um IAM ou AVC.

Apesar de ainda não estar estabelecido o impacto da vacinação contra influenza na redução de eventos e mortes por $\mathrm{DCbV} 38$, os benefícios da vacina e seu efeito protetor em relação a esses eventos cardiovasculares têm sido referidos na literatura 19,40,44,45. Uma metanálise recente mostrou que, em pacientes com DCV, a vacina pode reduzir mortalidade e eventos cardiovasculares combinados. Nos estudos de prevenção secundária, a mortalidade cardiovascular foi significativamente reduzida pela vacinação contra influenza ( $R R=0,45$, IC95\%: 0,26; 0,76, $p=0,003) 20$.

No presente estudo, pode-se observar maior redução dos coeficientes médios (interceptos dos modelos de regressão) de mortalidade por DCbV no período após o início das campanhas de vacinação. Outros estudos observacionais têm demonstrado associação entre a vacinação contra a influenza e a redução do risco de AVC 38,46,47. Lin et al. 45 identificaram, em estudo de caso-controle, que idosos vacinados apresentavam menor risco de internação por AVC (OR = 0,76, IC95\%: 0,60; 0,97) e que o risco diminuiu com o número crescente de vacinas (revacinação anual). Recentemente, uma revisão sistemática de estudos observacionais com metanálise também mostrou que a vacinação está associada a um risco menor de AVC, visto que indivíduos vacinados apresentaram menor risco em comparação aos indivíduos não vacinados ( $\mathrm{OR}=0,82$, IC95\%: 0,75; 0,91, $\mathrm{p}<0,001) 48$.

Estudo de coorte realizado no sul de Taiwan que acompanhou mais de 102 mil idosos ao longo de dez meses identificou que a vacina contra influenza esteve fortemente associada à redução da mortalidade dos idosos por causas específicas (como doenças cardíacas e AVC) e mortalidade por todas as causas 18. Em estudo de coorte realizado nos Estados Unidos, a vacinação também esteve associada à redução do risco de hospitalizações de idosos por DCbV, doenças cardíacas (DIC e insuficiência cardíaca congestiva), pneumonia, bem como redução do risco de morte por todas as causas 49 .

Além de identificar diferentes pontos de mudança na série considerada, a análise dos dados por meio do modelo joinpoint regression realizada neste estudo permitiu identificar a variação percentual ocorrida na mortalidade por DCbV no período. Particularmente para os idosos com idade entre 70-79 anos, estudos anteriores têm apontado maiores coberturas vacinais, desde o início das campanhas no país 50,51,52. Neste estudo, apesar de ter sido observada maior estimativa pontual para a redução de AAPC nesse subgrupo, essa redução não foi estatisticamente diferente daquelas observadas para as outras faixas etárias.

A análise joinpoint também mostrou que, especificamente para o total de idosos e para aqueles do sexo masculino com 60-69 anos, foram identificadas mudanças na tendência de mortalidade em 1998. Destaca-se que as campanhas de vacinação contra a influenza nos idosos tiveram início no Estado de São Paulo em 1998 e, no âmbito nacional, em 1999. Desde então, as campanhas são realizadas anualmente como estratégia para prevenção de quadros graves da influenza e dos riscos da doença em idosos e pacientes com DCV crônicas 12.

Considerando-se que a população idosa brasileira vem crescendo acima da média mundial e que as coberturas vacinais contra a influenza no grupo etário de 60 anos ou mais vêm aumentando no país (de 64,8\% no ano 2000 para 94,4\% em 2016; 7,5 milhões de doses aplicadas em 1999 para 20,8 milhões 
em 2016), parte dessa redução da mortalidade por DCbV pode ser reflexo de uma maior adesão dessa população à vacinação 12,15 .

Deve-se considerar a resposta imunológica mais fraca e demorada no idoso, e que anormalidades imunológicas em pacientes com doenças crônicas como diabetes, cardiopatias e doenças respiratórias crônicas piorem o prognóstico desses pacientes 53. Em pessoas mais idosas, a resposta imunológica pode ser menos eficaz na prevenção da doença (possivelmente devido à imunossenescência) 54 , no entanto a vacina pode reduzir a gravidade da influenza e a incidência de complicações e mortes $11,12,55,56$.

Diversos fatores podem influenciar o impacto das campanhas de vacinação, entre eles, as coberturas vacinais, a composição da vacina coincidente com os vírus circulantes, o momento oportuno das campanhas, além da variante viral predominante 57 . A circulação do vírus influenza A/H3N2 tem sido associada à maior morbidade e mortalidade em idosos em várias partes do mundo. Em 20032004 e 2004-2005, a variante influenza A/Fujian/411/02(H3N2) associou-se ao aumento de casos de pneumonia em várias partes do mundo 58. Em 2006, de acordo com a Secretaria de Vigilância em Saúde do Ministério da Saúde, a estirpe viral da influenza A/Wisconsin/67/2005-H3N2 circulou no Brasil e no Estado de São Paulo 59. Cabe destacar que essa estirpe não estava incluída na composição da vacina daquele ano, e isso pode ter contribuído para o aumento dos quadros infecciosos mais graves entre os idosos.

Vários estudos têm usado modelos de regressão linear e polinomial na avaliação de tendência antes e depois. O presente estudo, além de considerar essa técnica de análise, também aplicou o modelo joinpoint regression, que identifica o momento em torno do qual ocorrem mudanças na tendência e calcula a variação percentual anual dos coeficientes de mortalidade para cada período de tempo delimitado pelos pontos de inflexão da reta, método ainda pouco usado em estudos ecológicos de série temporal na análise do impacto de intervenções em saúde pública no Brasil.

Deve-se considerar que, nos últimos anos, ocorreram importantes mudanças em aspectos que se relacionam a vários desfechos em saúde, inclusive ao óbito por DCbV. A melhoria nas condições socioeconômicas da população, o controle dos fatores de risco (hipertensão arterial, dislipidemias, tabagismo, entre outros), a expansão do acesso aos serviços de saúde e ao uso de medicamentos, o diagnóstico preciso e tratamento em tempo oportuno, o acesso aos procedimentos de alta tecnologia e a evolução dos cuidados terapêuticos na prevenção terciária são determinantes na redução das mortes por DCbV 3,34,35,36,37, principalmente entre os mais longevos. Nesse sentido, a comparação das tendências antes e depois da vacinação contra influenza, bem como aquela definida pelo modelo joinpoint, deve ser realizada com cautela. Apesar da maior redução dos coeficientes de mortalidade por DCbV observada para o total dos idosos em torno do início das campanhas, os pontos de inflexão variaram conforme o sexo e as faixas etárias. Ressalta-se que a utilização da análise joinpoint possibilita a identificação de pontos de mudanças nas tendências e, também, a obtenção de informações sobre a variação percentual anual dos coeficientes de mortalidade ocorrida no período estudado. Destaca-se, ainda, que as retas ajustadas pelo joinpoint regression são conectadas entre si, considerando os últimos pontos de cada segmento 25 .

No período estudado (1980-2012), houve mudança na Classificação Internacional de Doenças (CID-9 para CID-10). Destaca-se que, nas análises de tendências de mortalidade por causas, deve-se considerar que, com a introdução de uma nova CID, podem ocorrer mudanças na localização dos códigos de algumas doenças, bem como pode ocorrer que outros códigos se desdobrem em vários, tornando-se código único, e tais mudanças podem alterar a magnitude da frequência de doenças 60 . Isso pode impactar no aumento ou diminuição das frequências de óbitos por algumas causas específicas. Para evitar possíveis efeitos da implementação da CID-10 na tendência de mortalidade por DCbV, foi aplicada a razão de comparabilidade nos óbitos 21 .

Mesmo que ainda haja controvérsias sobre o benefício da vacina contra influenza na prevenção de mortes por DCbV, deve-se considerar que tanto a influenza como as DCV são importantes causas de complicações e óbitos nos idosos, e vários estudos têm mostrado benefícios da vacina em relação ao AVC 38,45,46,47,48 e à doença arterial coronariana 61. Isso reforça a necessidade de incentivar a vacinação nesses grupos de risco para redução da morbidade e mortalidade relacionadas à doença e, consequentemente, em prol de uma melhoria da qualidade de vida dessa população. 


\section{Conclusão}

Os resultados deste estudo mostram tendência de queda da mortalidade por DCbV nos idosos residentes no Estado de São Paulo entre 1980 e 2012. Também apontam uma maior redução percentual anual dos coeficientes a partir de 1998, no entanto as variações observadas não permitem inferir que possa ser devido a um possível impacto das campanhas de vacinação. A despeito das variações observadas para a tendência dos coeficientes no período após a vacinação, deve-se considerar que a ampliação do acesso aos serviços de saúde e ao uso de medicamentos, as ações de promoção da saúde e prevenção de agravos, como a vacinação contra a influenza, a redução do tabagismo e melhoria nas condições socioeconômicas, têm tido impacto positivo na redução da mortalidade por DCbV. Os achados do presente estudo adicionam informações para o debate sobre o possível efeito das campanhas de vacinação na redução da mortalidade por DCbV na população idosa.

\section{Colaboradores}

A. G. M Bacurau participou da concepção e do delineamento do estudo, da coleta e da análise dos dados, da interpretação dos resultados, da redação do artigo e da aprovação da versão final a ser publicada. R. O. Ferraz colaborou na análise estatística dos dados e na elaboração dos resultados. M. R. Donalisio participou da redação do artigo e da revisão crítica relevante do conteúdo intelectual. P. M. S. B. Francisco orientou o trabalho, contribuiu na concepção e no delineamento do estudo, na redação do artigo e na revisão crítica relevante do conteúdo intelectual e na aprovação da versão final do artigo a ser publicada.

\section{Informações adicionais}

ORCID: Aldiane Gomes de Macedo Bacurau (0000-0002-6671-2284); Rosemeire de Olanda Ferraz (0000-0001-5796-946X); Maria Rita Donalisio (0000-0003-4457-9897); Priscila Maria Stolses Bergamo Francisco (0000-0001-7361-9961).

\section{Agradecimentos}

À Coordenação de Aperfeiçoamento de Pessoal de Nível Superior (Capes), pela bolsa de estudo concedida à A. G. M. Bacurau.

\section{Referências}

1. Miranda GMD, Mendes ACG, Silva ALA. Population aging in Brazil: current and future social challenges and consequences. Rev Bras Geriatr Gerontol 2016; 19:507-19.

2. Departamento de Análise de Situação em Saúde, Secretaria de Vigilância em Saúde, Ministério da Saúde. Saúde Brasil 2013: uma análise da situação de saúde e das doenças transmissíveis relacionadas à pobreza. Brasília: Ministério da Saúde; 2014.

3. Departamento de Análise de Situação em Saúde, Secretaria de Vigilância em Saúde, Ministério da Saúde. Plano de ações estratégicas para o enfrentamento das doenças crônicas não transmissíveis (DCNT) no Brasil 2011-2022. Brasília: Ministério da Saúde; 2011.

4. World Health Organization. Cardiovascular disease. http://www.who.int/cardiovascular_ diseases/en/ (acessado em 05/Abr/2017).

5. Affiune A. Envelhecimento cardiovascular. In: Freitas EV, Py L, Cançado FAX, Doll J, Gorzoni ML, organizadores. Tratado de geriatria e gerontologia. 2a Ed. Rio de Janeiro: Editora Guanabara Koogan; 2006. p. 396-401.

6. Lotufo PA. Doenças cardiovasculares no Brasil. In: Serrano Júnior CV, Timerman A, Stefanini E, organizadores. Tratado de cardiologia SOCESP. v. 1. 2a Ed. Barueri: Edições Manole; 2009. p. 7-16.

7. Silveira RE, Santos AS, Sousa MC, Monteiro TSA. Expenses related to hospital admissions for the elderly in Brazil: perspectives of a decade. Einstein (São Paulo) 2013; 11:514-20.

8. Cohen C, Simonsen L, Kang J, Miller M, McAnerney J, Blumberg L, et al. Elevated influenza-related excess mortality in South African elderly individuals, 1998-2005. Clin Infect Dis 2010; 51:1362-9.

9. Bricks LF, Carvalhanas TRMP, Domingues CMAS, Pereira SF, Bellei NCJ. Influenza em pacientes com doenças cardíacas crônicas: o que há de novo? J Health Biol Sci 2015; 3:16571. 
10. Fiore AE, Uyeki TM, Broder K, Finelli L, Euler GL, Singleton JA, et al. Prevention and control of influenza with vaccines: recommendations of the Advisory Committee on Immunization Practices (ACIP), 2010. MMWR Recomm Rep 2010; 59:1-62.

11. World Health Organization. Influenza (seasonal). (Fact Sheet, 211). http://www.who.int/ mediacentre/factsheets/fs211/en/ (acessado em 05/Abr/2017).

12. Coordenação Geral do Programa Nacional de Imunizações, Departamento de Vigilância Epidemiológica, Secretaria de Vigilância em Saúde, Ministério da Saúde. Campanha Nacional de Vacinação Contra a Influenza: informe técnico. 19a Ed. Brasília: Ministério da Saúde; 2017.

13. Smith Jr. SC, Benjamin EJ, Bonow RO, Braun LT, Creager MA, Franklin BA, et al. AHA/ACCF secondary prevention and risk reduction therapy for patients with coronary and other atherosclerotic vascular disease: 2011 update. A guideline from the American Heart Association and American College of Cardiology Foundation. Circulation 2011; 124:2458-73.

14. Bocchi EA, Marcondes-Braga FG, Ayub-Ferreira SM, Rohde LE, Oliveira WA, Almeida DR, et al. III Diretriz brasileira de insuficiência cardíaca crônica. Arq Bras Cardiol 2009; $92(6$ Suppl 1):1-71.

15. Centro de Vigilância Epidemiológica "Prof Alexandre Vranjac”, Coordenação dos Institutos de Pesquisa, Secretaria de Estado da Saúde. Informe técnico da campanha nacional de vacinação para o idoso - 2002. ftp://ftp.cve.sau de.sp.gov.br/doc_tec/imuni/inf_tec_idoso02. pdf (acessado em 05/Abr/2017).

16. Francisco PMSB, Donalisio MR, Marín-León L. Trends in mortality from respiratory diseases among the elderly and the influenza vaccine intervention, 1980-2009. Rev Panam Salud Pública 2013; 34:155-61.

17. Luna EJA, Gattás VL, Campos SRSLC. Efetividade da estratégia brasileira de vacinação contra influenza: uma revisão sistemática. Epidemiol Serv Saúde 2014; 23:559-76.

18. Wang CS, Wang ST, Lai CT, Lin LJ, Chou P. Impact of influenza vaccination on major cause-specific mortality. Vaccine 2007; 25:1196-203.

19. Phrommintikul A, Kuanprasert S, Wongcharoen W, Kanjanavanit R, Chaiwarith R, Sukonthasarn A. Influenza vaccination reduces cardiovascular events in patients with acute coronary syndrome. Eur Heart J 2011; 32:1730-5.

20. Clar C, Oseni Z, Flowers N, Keshtkar-Jahromi $\mathrm{M}$, Rees K. Influenza vaccines for preventing cardiovascular disease. Cochrane Database Syst Rev 2015; (5):CD005050.

21. Anderson RN, Miniño AM, Hoyert DL, Rosenberg HM. Comparability of cause of death between ICD-9 and ICD-10: preliminary estimates. Natl Vital Stat Rep 2001; 49:1-32.
22. Neter J, Wasserman W, Kutner MH. Polynomial regression. In: Neter J, Wasserman W, Kutner MH, editors. Applied linear statistical models: regression, analysis of variance and experimental designs. 3rd Ed. Boston: Irwin; 1990. p. 315-41.

23. Latorre MRDO, Cardoso MRA. Análise de séries temporais em epidemiologia: uma introdução sobre os aspectos metodológicos. Rev Bras Epidemiol 2001; 4:145-52.

24. Charnet R, Freire CAL, Charnet EMR, Bonvino H. Análise de modelos de regressão linear: com aplicações. Campinas: Editora da Unicamp; 2008.

25. Kim HJ, Fay MP, Feuer EJ, Midthune DN. Permutation tests for Joinpoint regression with applications to cancer rates. Stat Med 2000; 19:335-51.

26. Division of Cancer Control \& Population Sciences, National Cancer Institute. Joinpoint help manual 4.3.1.0. https://surveillance.can cer.gov/joinpoint/Joinpoint_Help_4.3.1.0.pdf (acessado em 12/Mai/2016).

27. Shapiro SS, Wilk MB. An analysis of variance test for normality (complete samples). Biometrika 1965; 52:591-611.

28. White H. A heteroskedasticity-consistent covariance matrix estimator and a direct test for heteroskedasticity. Econometrica 1980; 48:817-38.

29. Godfrey LG. Testing against general autoregressive and moving average error models when the regressors include lagged dependent variables. Econometrica 1978; 46:1293-301.

30. Hastings WK. Monte Carlo sampling methods using Markov chains and their applications. Biometrika 1970; 57:97-109.

31. Lerman PM. Fitting segmented regression models by grid search. Appl Stat 1980; 29:7784.

32. Guimarães RM, Andrade SSCA, Machado EL, Bahia CA, Oliveira MM, Jacques FVL. Diferenças regionais na transição da mortalidade por doenças cardiovasculares no Brasil, 1980 a 2012. Rev Panam Salud Pública 2015; 37:83-9.

33. Mansur AP, Favarato D. Trends in mortality rate from cardiovascular disease in Brazil, 1980-2012. Arq Bras Cardiol 2016; 107:20-5.

34. Yusuf S, Hawken S, Ounpuu S, Dans T, Avezum A, Lanas F, et al. Effect of potentially modifiable risk factors associated with myocardial infarction in 52 countries (the INTERHEART study): case-control study. Lancet 2004; 364:937-52.

35. Souza MFM, Alencar AP, Malta DC, Moura L, Mansur AP. Serial temporal analysis of ischemic heart disease and stroke death risk in 5 regions of Brazil from 1981 to 2001. Arq Bras Cardiol 2006; 87:735-40.

36. Schmidt MI, Duncan BB, Azevedo e Silva G, Menezes AM, Monteiro CA, Barreto SM, et al Chronic non communicable diseases in Brazil: burden and current challenges. Lancet 2011; 377:1949-61. 
37. Duncan BB, Chor D, Aquino EML, Bensenor IM, Mill JG, Schmidt MI, et al. Doenças crônicas não transmissíveis no Brasil: prioridade para enfrentamento e investigação. Rev Saúde Pública 2012; 46 Suppl 1:126-34.

38. Mansur AP, Favarato D, Ramires JAF. Vacina contra o vírus da influenza e mortalidade por doenças cardiovasculares na cidade de São Paulo. Arq Bras Cardiol 2009; 93:395-9.

39. Madjid M, Aboshady I, Awan I, Litovsky S, Casscells SW. Influenza and cardiovascular disease: is there a causal relationship? Tex Heart Inst J 2004; 31:4-13.

40. Warren-Gash C, Smeeth L, Hayward AC. Influenza as a trigger for acute myocardial infarction or death from cardiovascular disease: a systematic review. Lancet Infect Dis 2009; 9:601-10.

41. Nunes B, Viboud C, Machado A, Ringholz C, Rebelo-de-Andrade H, Nogueira P, et al. Excess mortality associated with influenza epidemics in Portugal, 1980 to 2004. PLoS One 2011; 6:e20661.

42. Madjid M, Miller CC, Zarubaev VV, Marinich IG, Kiselev OI, Lobzin YV, et al. Influenza epidemics and acute respiratory disease activity are associated with a surge in autopsy-confirmed coronary heart disease death: results from 8 years of autopsies in 34,892 subjects. Eur Heart J 2007; 28:1205-10.

43. Naghavi M, Barlas Z, Siadaty S, Naguib S, Madjid M, Casscells W. Association of influenza vaccination and reduced risk of recurrent myocardial infarction. Circulation 2000; 102:3039-45.

44. Udell JA, Zawi R, Bhatt DL, Keshtkar-Jahromi M, Gaughran F, Phrommintikul A, et al. Association between influenza vaccination and cardiovascular outcomes in high-risk patients: a meta-analysis. JAMA 2013; 310:1711-20.

45. Lin H-C, Chiu H-F, Ho S-C, Yang C-Y. Association of influenza vaccination and reduced risk of stroke hospitalization among the elderly: a population-based case-control study. Int J Environ Res Public Health 2014; 11:3639-49.

46. Lavallée P, Perchaud V, Gautier-Bertrand M, Grabli D, Amarenco P. Association between influenza vaccination and reduced risk of brain infarction. Stroke 2002; 33:513-8.

47. Grau AJ, Fischer B, Barth C, Ling P, Lichy C, Buggle F. Influenza vaccination is associated with a reduced risk of stroke. Stroke 2005; 36:1501-6.

48. Lee KR, Bae JH, Hwang IC, Kim KK, Suh HS, Ko KD. Effect of influenza vaccination on risk of stroke: a systematic review and meta-analysis. Neuroepidemiology 2017; 48:103-10.

49. Nichol KL, Nordin J, Mullooly J, Lask R, Fillbrandt $\mathrm{K}$, Iwane $\mathrm{M}$. Influenza vaccination and reduction in hospitalizations for cardiac disease and stroke among the elderly. $\mathrm{N}$ Engl J Med 2003; 348:1322-32.
50. Francisco PMSB, Donalisio MR, Barros MBA, Cesar CLG, Carandina L, Goldbaum M. Vacinação contra influenza em idosos por área de residência: prevalência e fatores associados. Rev Bras Epidemiol 2006; 9:162-71.

51. Campos EC, Sudan LCP, Mattos ED, Fidelis R. Fatores relacionados à vacinação contra a gripe em idosos: estudo transversal, Cambé, Paraná, Brasil. Cad Saúde Pública 2012; 28:878-88.

52. Moura RF, Andrade FB, Duarte YAO, Lebrão ML, Antunes JLF. Fatores associados à adesão à vacinação anti-influenza em idosos não institucionalizados, São Paulo, Brasil. Cad Saúde Pública 2015; 31:2157-68.

53. Thompson WW, Shay DK, Weintraub E, Brammer L, Cox N, Anderson LJ, et al. Mortality associated with influenza and respiratory syncytial virus in the United States. JAMA 2003; 289:179-86

54. Veiga AMV. Imunidade e envelhecimento. In: Freitas EV, Py L, Cançado FAX, Doll J, Gorzoni ML, organizadores. Tratado de geriatria e gerontologia. 2a Ed. Rio de Janeiro: Editora Guanabara Koogan; 2006. p. 846-54.

55. Nichol KL, Margolis KL, Wuorenma J, Von Sternberg T. The efficacy and cost effectiveness of vaccination against influenza among elderly persons living in the community. N Engl J Med 1994; 331:778-84.

56. Vu T, Farish S, Jenkins M, Kelly H. A metaanalysis of effectiveness of influenza vaccine in persons aged 65 years and over living in the community. Vaccine 2002; 20:1831-6.

57. Centers for Disease Control and Prevention. Efectividad de la vacuna contra la influenza: Preguntas y respuestas para profesionales de la salud. https://espanol.cdc.gov/enes/flu/ professionals/vaccination/effectivenessqa.htm (acessado em 10/Ago/2017).

58. Russell CA, Jones TC, Barr IG, Cox NJ, Garten RJ, Gregory V, et al. The global circulation of seasonal influenza A (H3N2) viruses. Science 2008; 320:340-6.

59. Centro de Vigilância Epidemiológica "Prof. Alexandre Vranjac”, Coordenação dos Institutos de Pesquisa, Secretaria de Estado da Saúde. Informe técnico da campanha nacional de vacinação para o idoso - 2007. ftp://ftp.cve. saude.sp.gov.br/doc_tec/imuni/if_idoso07.pdf (acessado em 10/Ago/2017).

60. Grassi PR, Laurenti R. Implicações da introdução da 10a revisão da Classificação Internacional de Doenças em análise de tendência da mortalidade por causas. Inf Epidemiol SUS 1998; 7:43-7.

61. MacIntyre CR, Mahimbo A, Moa AM, Barnes $M$. Influenza vaccine as a coronary intervention for prevention of myocardial infarction. Heart 2016; 102:1953-6. 


\section{Abstract}

This study describes the trend of the coefficients of mortality due to cerebrovascular diseases (CbVD) among the elderly in São Paulo State, Brazil, from 1980 to 2012, before and after influenza vaccination campaigns, and identifies change points. It is an ecological, time-series study carried out with death data from the Health Ministry's Mortality Information System and population data from the Brazilian Institute of Geography and Statistics. We used linear, polynomial and joinpoint regression models in the data analysis. Between 1980 and 2012, there were 480,955 deaths due to $\mathrm{Cb}$ $V D$. The mean mortality coefficients decreased for both sexes in all age groups we analyzed, with the greatest reduction in the older ages and male sex. We observed a significant reduction in the mortality trend in 1998 for male sex in the age group 60-69 years (annual percent change $-A P C=$ $-3 \%, 95 \%$ CI: $-4.3 ;-1.6)$ and for all elderly $(A P C=$ $-3.8 \%, 95 \%$ CI: $-4.4 ;-3.1)$. Considering the period as a whole, we did not observe change points for the age group 70-79 (average annual percent change $-A A P C=-3.3 \%$, 95\%CI: $-3.5 ;-3.1)$ and, for male sex, for the group $\geq 80$ years $(A A P C=-2.9 \%$, 95\%CI: - 3.1; - 2.6). For all elderly, the mean percentage reduction was of $3.1 \%$ per year $(A A P C=$ $-3.1 \%, 95 \%$ CI: -3.5; -2.7). Results show a reduction in the mortality due to CbVD in the period, with different percentage variations in coefficient reduction. The study's findings add information to the debate regarding possible effects of vaccination campaigns in reducing mortality due to CbVD among the elderly.

Cardiovascular Diseases; Cerebrovascular Disorders; Influenza Vaccines; Aged; Mortality

\section{Resumen}

Este estudio describe la tendencia de los coeficientes de mortalidad por enfermedades cerebrovasculares (DCbV) en ancianos del Estado de São Paulo, Brasil, entre 1980 y 2012, antes y después de las campañas de vacunación contra la gripe, $e$ identifica puntos de cambio. Se trata de un estudio ecológico de serie temporal, realizado con datos de óbitos del Sistema de Informaciones sobre Mortalidad del Ministerio de la Salud y datos poblacionales del Instituto Brasileño de Geografía y Estadística. Para el análisis de los datos se utilizaron modelos de regresión lineal, polinomial y regresión joinpoint. Entre 1980 y 2012, se registraron 480.955 óbitos por DCbV. Los coeficientes medios de mortalidad disminuyeron en ambos sexos, en todas las franjas de edad analizadas, con una mayor reducción en las edades más longevas y dentro del sexo masculino. Se observó una caída significativa en la tendencia de la mortalidad en 1998 en el sexo masculino, en la franja de 60-69 años (annual percent change $-A P C=-3 \%$, IC95\%: $-4,3 ;-1,6)$ y para el total de los ancianos $(A P C=$ -3,8\%, IC95\%: -4,4; -3,1). Considerándose el período como un todo, no se observaron puntos de cambios para la franja de 70-79 (average annual percent change - AAPC $=-3,3 \%$, IC95\%: $-3,5$; $-3,1)$ y en el sexo masculino para el grupo ? 80 años (AAPC =-2,9\%, IC95\%: -3, 1;-2,6). Para el total de ancianos, la reducción del porcentaje medio fue $3,1 \%$ al año $(A A P C=-3,1 \%$, IC95\%: $-3,5$; $-2,7)$. Los resultados mostraron la reducción de la mortalidad por $D C b V$ en el período estudiado, con diferentes variaciones porcentuales de caída de los coeficientes. Los hallazgos de este estudio añaden información para el debate sobre el posible efecto de las campañas de vacunación en la reducción de la mortalidad por $D C b V$ dentro de la población anciana.

Enfermedades Cardiovasculares; Trastornos Cerebrovasculares; Vacunas Contra la Influenza; Anciano; Mortalidad
Recebido em 21/Ago/2017

Versão final reapresentada em 11/Out/2018 Aprovado em 25/Out/2018 